\title{
Numerical simulation of mold filling of semi-solid slurry based on viscosity theory
}

\author{
Qiuping Wang*, Jing Xu, Baigong Wu, and Jiayang Gu \\ Marine Equipment technology Institute, Jiangsu University of Science and Techology, Zhenjiang, \\ Jiangsu, 212003, P.R. China
}

\begin{abstract}
The mold filling of semi-solid slurry involves intricate theory and physical phenomenon. The influence of inner gate shape and filling speed on free surface and liquid-solid distribution is investigated by adopting finite element numerical simulation. The effect of viscosity is considered in the modelling. The results show that the inner gate shape affects the free surface. The filling speed of $3 \mathrm{~m} / \mathrm{s}$ is favorable for the uniform distribution of solid-liquid phases. It has important guiding significance for the optimization of semi-solid forming process and molding design.
\end{abstract}

Keywords: Semi-solid slurry, Mold filling, Numerical simulation, Gate shape, Filling speed.

\section{Introduction}

Semi-solid formed castings have the advantages of compact microstructure and excellent mechanical properties, which have been widely applied in automobile, aircraft, electronics and other industries[1-3]. However, if the mold design and the parameters options of filling process are unreasonable during semi-solid die casting, it would lead to defects, such as insufficient pouring, cold shut and shrinkage porosity. Therefore, it is the key to the production of high quality gradually through designing an excellent casting system and reasonable mold filling[4].

The aluminum alloy has been widely discussed in many references for semi-solid forming $[5,6]$. The semi-solid mold filling of aluminum alloy is a very complex process, which involves fluid viscosity, heat transfer, computer graphics, calculation method, mathematical theory of partial differential equation and casting process theory[7]. The changes of solid and liquid fraction in semi-solid slurry usually affect the flow characteristics in the slurry forming process, and the melt viscosity will impact the alloy formability. From the view of numerical simulation of forming process, a suitable rheological model of semi-solid slurry being established will directly affect the accuracy of simulation results. This paper makes a full consideration with the viscosity changes of semi-solid slurry. The mold filling process of semi-solid slurry is simulated and analyzed by utilizing finite element numerical simulation.

\footnotetext{
* Corresponding author: a21096@163.com
} 


\section{Modelling}

\subsection{Materials parameters}

The high iron (2wt \%) and high silicon $(25 \mathrm{wt} \%)$ aluminum alloy is used in this paper. The thermal conductivity, density, enthalpy and solid phase fraction are shown in Fig. 1. The liquidus and the solidus temperature are $752{ }^{\circ} \mathrm{C}$ and $575{ }^{\circ} \mathrm{C}$, respectively.

(a)

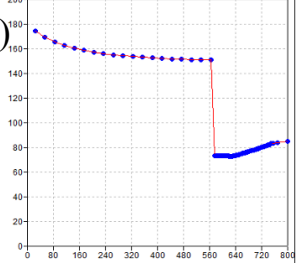

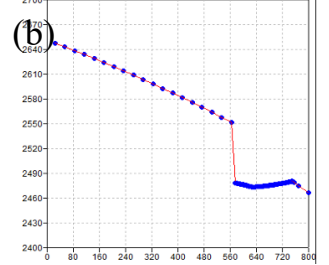
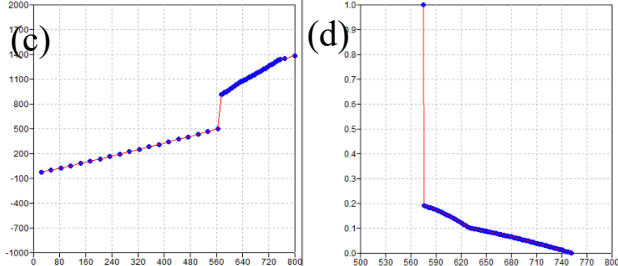

Fig. 1. The curves versus temperatures, (a) thermal conductivity, (b) density, (c) enthalpy, (d) solid fraction.

\subsection{Mathematical model}

Mold filling process is a non constant temperature flow process accompanied by heat loss and solidification. The whole filling process should follow many laws, such as momentum conservation, mass conservation and energy conservation. In this study, momentum equation, continuity equation and energy equation are used to express the filling process.

\subsubsection{Mass conservation equation}

The continuity equation is a mathematical expression of mass conservation law, which is suitable for both ideal and viscous fluids. The equation is expressed as follows:

$$
\frac{\partial \rho}{\partial t}+\nabla(\rho V)=0
$$

where $\rho$ is the fluid density, $V$ is the fluid velocity, $t$ is time.

\subsubsection{Momentum conservation equation}

The Navier-Stokes equation is mathematical expression of momentum conservation law, which can be expressed by:

$$
\begin{aligned}
& \rho\left(\frac{\partial u}{\partial t}+u \frac{\partial u}{\partial x}+v \frac{\partial u}{\partial y}+w \frac{\partial u}{\partial z}\right)=-\frac{\partial p}{\partial x}+\rho g x+\mu\left(\frac{\partial^{2} u}{\partial x^{2}}+\frac{\partial^{2} u}{\partial y^{2}}+\frac{\partial^{2} u}{\partial z^{2}}\right) \\
& \rho\left(\frac{\partial v}{\partial t}+u \frac{\partial v}{\partial x}+v \frac{\partial v}{\partial y}+w \frac{\partial v}{\partial z}\right)=-\frac{\partial p}{\partial y}+\rho g y+\mu\left(\frac{\partial^{2} v}{\partial x^{2}}+\frac{\partial^{2} v}{\partial y^{2}}+\frac{\partial^{2} v}{\partial z^{2}}\right) \\
& \rho\left(\frac{\partial w}{\partial t}+u \frac{\partial w}{\partial x}+v \frac{\partial w}{\partial y}+w \frac{\partial w}{\partial z}\right)=-\frac{\partial p}{\partial z}+\rho g z+\mu\left(\frac{\partial^{2} w}{\partial x^{2}}+\frac{\partial^{2} w}{\partial y^{2}}+\frac{\partial^{2} w}{\partial z^{2}}\right)
\end{aligned}
$$

\subsubsection{Energy conservation equation}

According to the Fourier's law of heat conduction and the energy conservation principle, the equation is obtained as follows: 


$$
\frac{\partial\left(\rho c_{p} T\right)}{\partial t}+\frac{\partial\left(\rho c_{p} T u\right)}{\partial x}+\frac{\partial\left(\rho c_{p} T v\right)}{\partial y}+\frac{\partial\left(\rho c_{p} T w\right)}{\partial z}=\frac{\partial}{\partial x}\left(\lambda \frac{\partial T}{\partial x}\right)+\frac{\partial}{\partial y}\left(\lambda \frac{\partial T}{\partial y}\right)+\frac{\partial}{\partial z}\left(\lambda \frac{\partial T}{\partial z}\right)
$$

\subsubsection{Semi-solid model}

When the semi-solid alloy melt is injected into the die cavity, the viscosity changes with the change of shear rate. When the shear rate is large, the dendrites are interrupted. The viscosity of the semi-solid alloy melt entering the mold will decrease and the fluidity will increase.

$$
\begin{gathered}
\mu(\dot{\gamma}, T)=\mu_{0}(T) \dot{\gamma}_{0}{ }^{n(T)} \quad \dot{\gamma} \leq \dot{\gamma}_{0} \\
\mu(\dot{\gamma}, T)=\mu_{0}(T) \dot{\gamma}^{n(T)} \\
\dot{\gamma}=\sqrt{\left\{\left(\frac{\partial \mathbf{u}}{\partial \mathbf{y}}\right)^{2}+\left(\frac{\partial \mathbf{w}}{\partial \mathbf{y}}\right)^{2}+\left(\frac{\partial \mathbf{v}}{\partial \mathbf{z}}\right)^{2}+\left(\frac{\partial \mathbf{u}}{\partial \mathbf{z}}\right)^{2}+\left(\frac{\partial \mathbf{v}}{\partial \mathbf{x}}\right)^{2}+\left(\frac{\partial \mathbf{w}}{\partial \mathbf{x}}\right)^{2}\right\}}
\end{gathered}
$$

where $\mu_{0}$ is the maximum viscosity value, $K$ is the influence coefficient of shear rate change on viscous force, $n$ is the variation law of viscous force, $u, v$ and $w$ are velocity components in $\mathrm{x}, \mathrm{y}$ and $\mathrm{z}$ coordinate directions respectively.

\subsubsection{Heat transfer equation}

During solidification heat conduction, Fourier heat conduction partial differential equation is used to describe the relationship between temperature, time and space. The equation is as follows:

$$
\rho C \frac{\partial T}{\partial t}+f_{L} \rho C\left(u \frac{\partial T}{\partial x}+v \frac{\partial T}{\partial y}+w \frac{\partial T}{\partial z}\right)=\lambda\left(\frac{\partial^{2} T}{\partial x^{2}}+\frac{\partial^{2} T}{\partial y^{2}}+\frac{\partial^{2} T}{\partial z^{2}}\right)+Q
$$

When the latent heat release, temperature gradient and liquid phase rate in the two-phase region are small, which can be ignored, the above equation can be simplified to:

$$
\frac{\partial T}{\partial t}=\frac{\lambda}{c \rho}\left(\frac{\partial^{2} T}{\partial x^{2}}+\frac{\partial^{2} T}{\partial y^{2}}+\frac{\partial^{2} T}{\partial z^{2}}\right)-\frac{Q}{\rho C}
$$

\subsubsection{Initial condition}

The initial condition is the temperature filed distribution at $\mathrm{t}=0$, which can be expressed:

$$
\left.T\right|_{t=0}=\varphi(x, y)
$$

\subsubsection{Boundary conditions}

The interface between casting and mold is the boundary. The heat flux q passing through the interface is expressed:

$$
q=h\left(T_{1}-T_{2}\right)
$$


where $h$ is the interfacial heat transfer coefficient, $\underline{T}_{\underline{l}}$ is the temperature on one side of the interface, $T_{2}$ is on the other side. The equivalent heat transfer coefficient $\lambda_{e}$ of the adjacent nodes on both sides of the interface is expressed:

$$
\frac{\Delta x}{\lambda_{e}}=\frac{\Delta x / 2}{\lambda_{1}}+\frac{\Delta x / 2}{\lambda_{2}}+\frac{1}{h}
$$

where $\Delta x$ is the cell width, $\lambda_{1}$ is the thermal conductivity on one side of the interface, $\lambda_{2}$ is the other side.

\subsection{Meshing}

According to the above mathematical model, the effects of gate shape and filling speed on the filling process of semi-solid slurry are simulated respectively. The three-dimensional solid modeling was meshed with ProEngineering and imported into ProCAST software. Two kinds of cylinder liner model are established in Fig. 2(a) and (c), the corresponding meshing are showed in Fig. 2(b) and (d). The whole model is composed of concave die, fixed die, core with radian and casting mold. The minimum unit of the grid is $5 \mathrm{~mm}$.

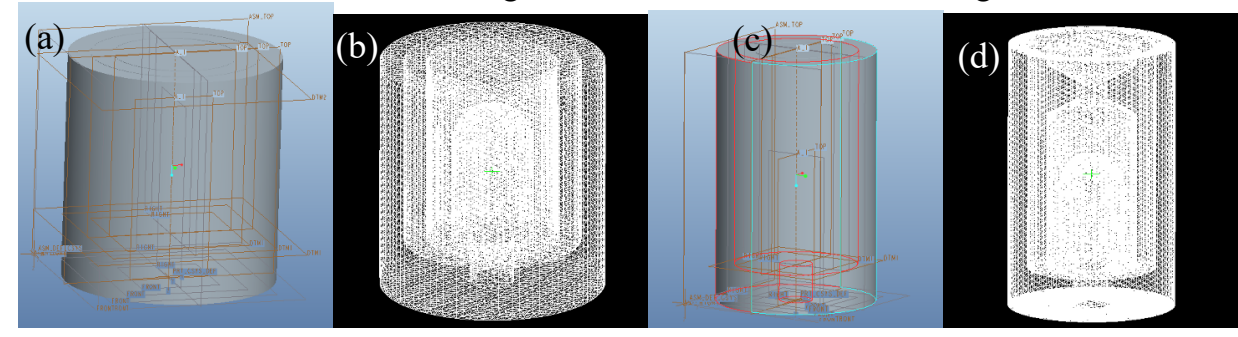

Fig. 2. (a), (c) Cylinder liner model, (b), (d) volume mesh.

\section{Results}

\subsection{Influence of inner gate shape}

Figures 3 shows the influence of inner gate shape on free surface morphology at the front of mold filling flow of semi-solid slurry. The mold filling temperature, filling speed and pressure are set as $580{ }^{\circ} \mathrm{C}, 5 \mathrm{~m} / \mathrm{s}$ and $25 \mathrm{MPa}$ respectively. As seen in Figs. 3(a) - (c), the mold filling front of semi-solid slurry is almost in the same horizontal plane, and the free surface morphology changes little during the whole process. When the inner gate shape changes, the free surface occurs to vary. As shown in Figs. 3(d) - (f), the semi-solid slurry flow down the core surface in jet form. Combined with Fig. 4(e), when the semi-solid slurry flows to the position where the inner gate shape changes, the flow speed suddenly increases from the original $5 \mathrm{~m} / \mathrm{s}$ to $10 \mathrm{~m} / \mathrm{s}$, so that the speed of semi-solid slurry increases when flowing to the core (as seen in Figs. 4 (c) and (g)). The free surface profile no longer keeps the same horizontal plane and moves forward in parallel. While the pilot fluid is formed to push forward. 


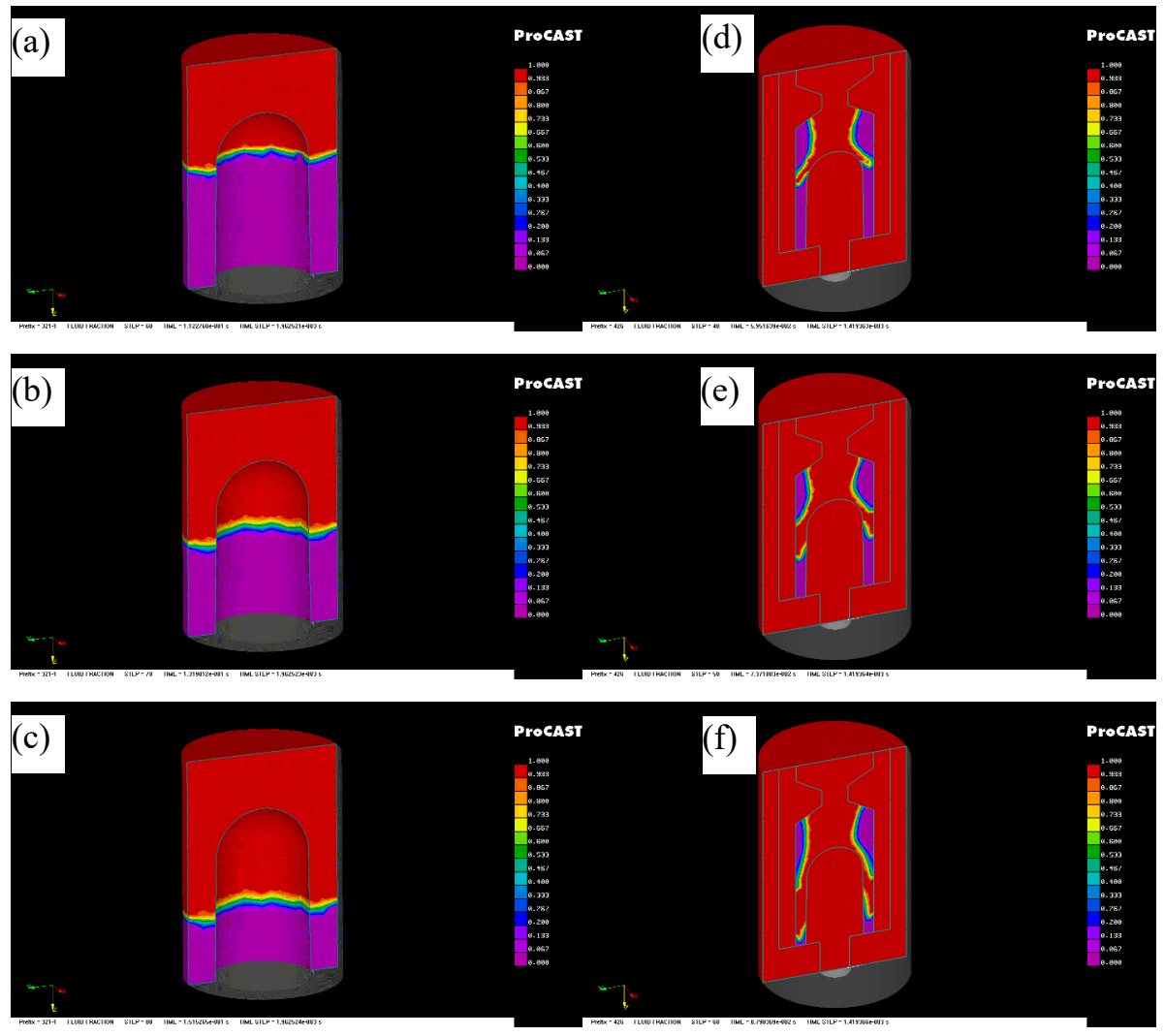

(a) - (c) is the first kind of cylinder liner model, (d) - (e) is the second kind of cylinder liner model

Fig. 3. Free surface during mold filling at $60,70,80$ steps respectively.

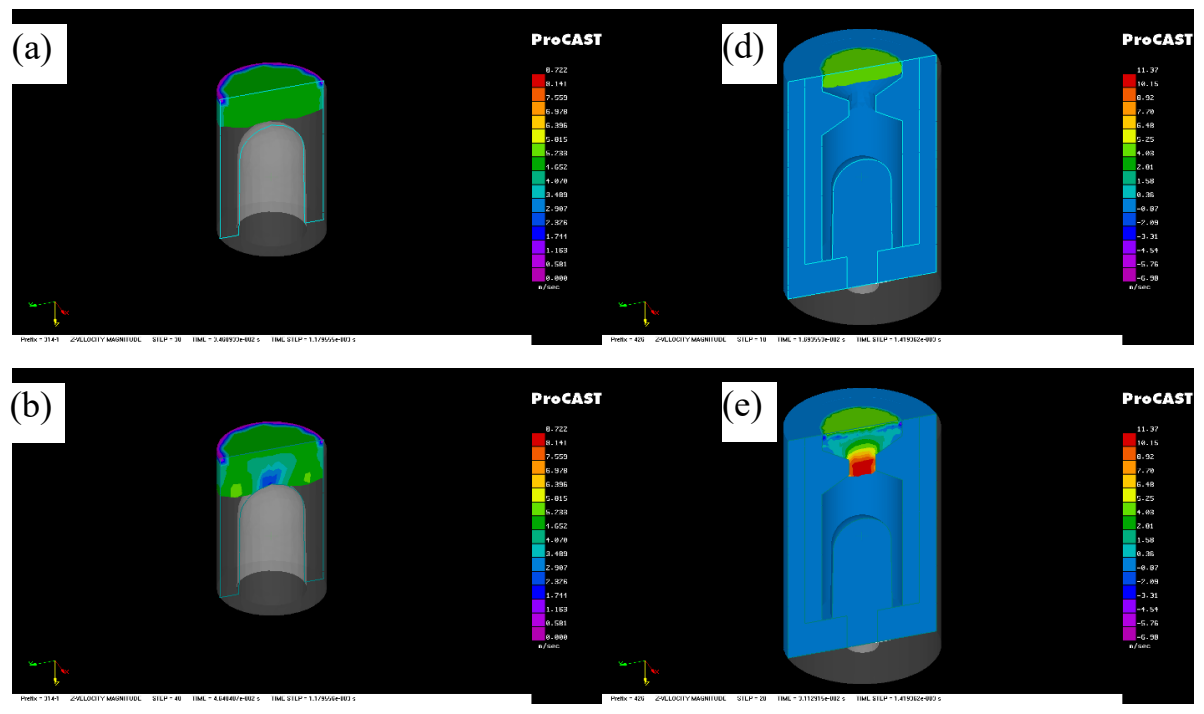




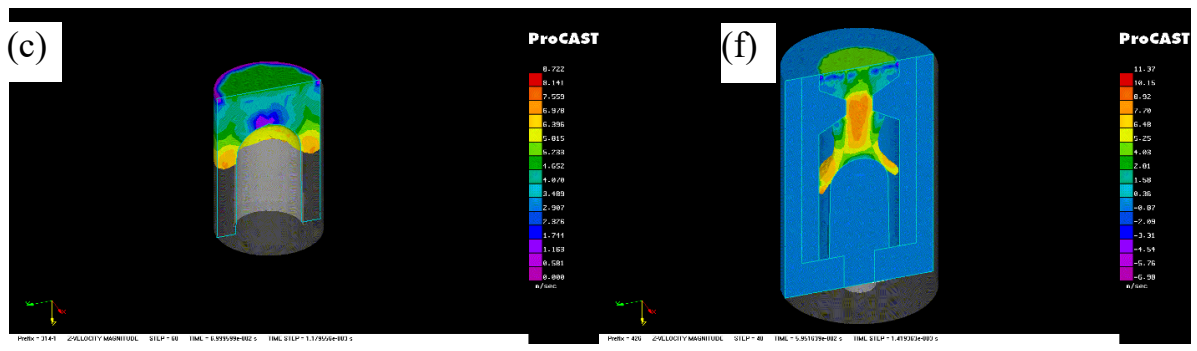

(a) - (c) is the first kind of cylinder liner model, (d) - (e) is the second kind of cylinder liner model

Fig. 4. Speed during mold filling.

\subsection{Influence of mold filling speed}

When the mold filling speed is $5 \mathrm{~m} / \mathrm{s}$, the power of liquid phase wrapping solid phase movement is greater due to the large mold filling speed, as seen in Fig. 5(a). The solid-liquid phase distribution is more uniform and there is no obvious solid-liquid separation. As the mold filling speed is $3 \mathrm{~m} / \mathrm{s}$, the apparent viscosity in the filling process shows an obvious gradient distribution, and the solid-liquid separation is ideal (see Fig. 5(b)). When the mold filling speed is set as $1 \mathrm{~m} / \mathrm{s}$, the liquid phase does not have enough power to wrap the solid phase and move together due to the slow filling speed. As seen in Fig. 5(c), large block segregation and serious liquid-solid separation occur, which is not conductive to the formation of gradient distribution.
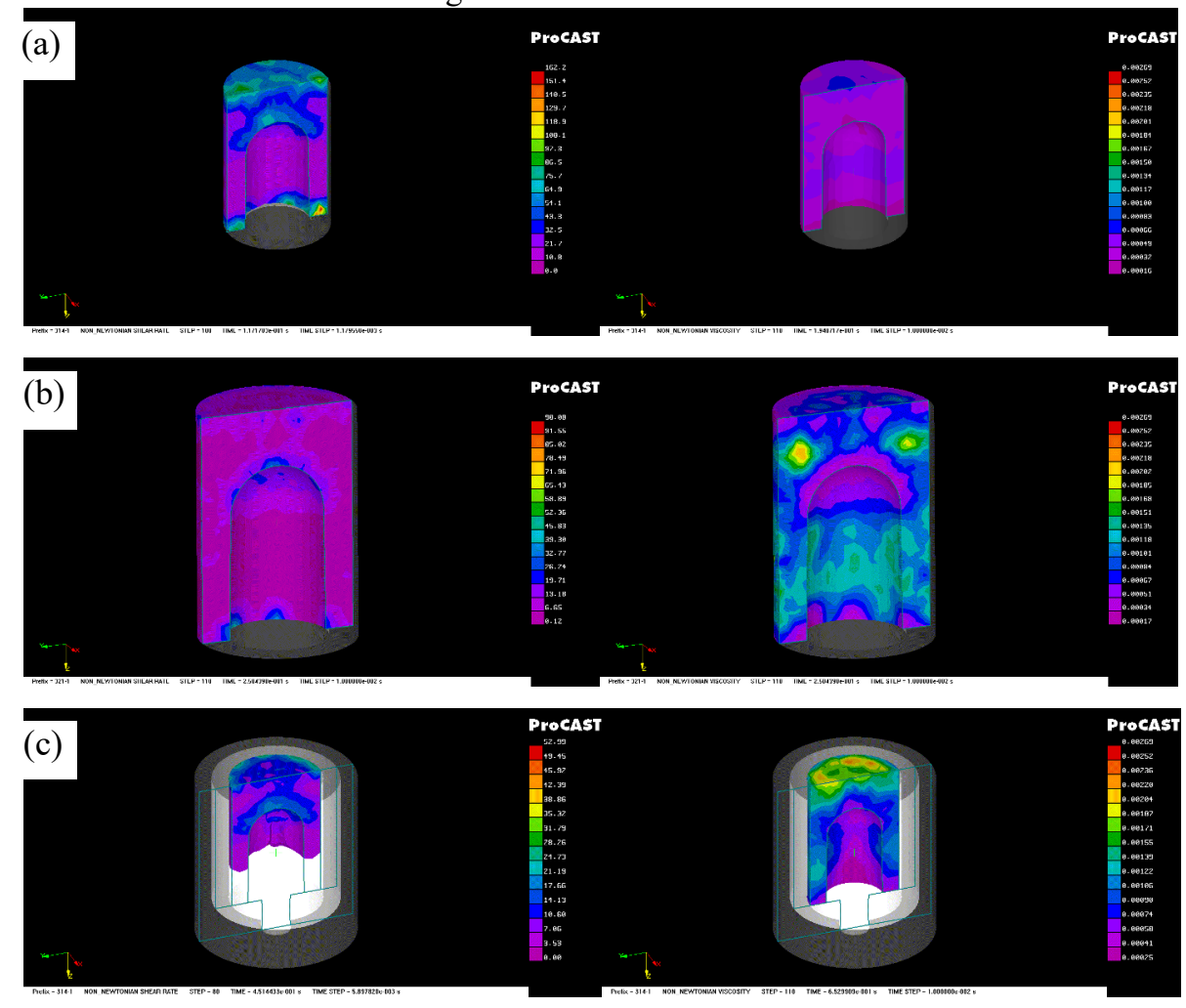

Fig. 5. Shear rate (left) and apparent viscosity (right) of non-Newtonian fluid in semi-solid slurry The mold filling speed (a) $5 \mathrm{~m} / \mathrm{s}$, (b) $3 \mathrm{~m} / \mathrm{s}$, (c) $1 \mathrm{~m} / \mathrm{s}$ 


\section{Conclusion}

This paper makes an investigation on the mold filling simulation of aluminum alloy semi-solid slurry. The finite element modelling results based on viscosity theory indicate that inner gate shape change affects the flow, so that the free surface varies. Faster or lower filling speed is not suitable for achieving uniform liquid-solid distribution.

The author would like to acknowledge the support from the Self-cultivation Project of Jiangsu University High Tech Ship Collaborative Innovation Center (HZ20200006)

\section{References}

1. A Kolahdooz, SA Dehkordi 2019 Effects of important parameters in the production of Al-A356 alloy by semi-solid forming process. Journal of Materials Research and Technology vol 8 p 189-198

2. Y Qiao, YU Huping, JM Zhang, Y Shen 2019 A new technology of microforming - Research progress on semi-solid microforming. Journal of Plasticity Engineering vol 26

3. GF Xiao, JF Jiang, Y Wang, YZ Liu 2019 Research progress of semi-solid processing technology of high-melting alloy. Journal of Plasticity Engineering vol 26

4. HH Zhang, GJ Shao, LP Xu 2003 Computer simulation of filling complex cavity during semi-solid die casting. Foundry vol 52 p 769-772

5. W Mao, Y Bai, G Tang 2006 Preparation for Semi-Solid Aluminum Alloy Slurry under Weak Electromagnetic Stirring Conditions. Journal of Materials Science and Technology, vol 24 p 447-451

6. G Wang, B Zhou, XU Chun, Z Wang 2019 Preparation of Semi-solid Microstructure of A356 Aluminum Alloy by Enhanced-cooling Mixing[J]. Hot Working Technology vol 48

7. G Chen, S Zheng, B Wang 2019 Research progress of numerical simulation technology for semi-solid forming. Ordance Materials Science and Engineering vol 42 